\title{
Intersubband relaxation of two-dimensional electrons in heterostructures
}

\author{
Vladimir I. Fal'ko* \\ Max-Planck-Institut für Festkorperforschung, Heisenbergstrasse 1, 7000 Stuttgart 80, Germany
}

(Received 17 November 1992)

\begin{abstract}
We calculate the lifetime of a nonequilibrium electron in the first excited subband in the lowdensity heterostructure where this photocreated carrier occurs at the last stage of its cooling. The electron interaction with acoustic phonons gives the dominant intersubband relaxation mechanism, if the intersubband energy splitting and the Fermi energy splitting are relatively small, $1>\epsilon_{F} / \Delta_{10}>$ 0.7-0.8. In $\mathrm{GaAs}-\mathrm{Al}_{x} \mathrm{Ga}_{1-x} \mathrm{As}$ heterostructures the intersubband relaxation determines the excitedelectron lifetime to be of the order of $\tau_{\text {phon }} \sim$ nanoseconds which depends slightly on the value of the two-dimensional electron density. When the ratio $\epsilon_{F} / \Delta_{10}$ is smaller, the intersubband relaxation is determined by the Auger-like electron-electron scattering whose rate can increase up to the value $\tau_{\text {Aug }}^{-1} \sim 10^{10} \sec ^{-1}$.
\end{abstract}

\section{INTRODUCTION}

Optical studies of two-dimensional (2D) electron systems ${ }^{1}$ have been extended during recent years to the investigation of the recombination in high-quality heterostructures at low temperatures. ${ }^{2}$ After identification in earlier works $\mathrm{ks}^{2-4}$ of the spectral lines associated with the recombination of $2 \mathrm{D}$ electrons with free and acceptor bound holes, the kinetics of luminescence and related relaxation phenomena became the subject of more recent experimental activities. ${ }^{5,6}$ Among these observations one can find those where the kinetic phenomena manifested themselves indirectly through the interplay of intensities of different spectral lines ${ }^{5}$ and also the direct time-resolved optical measurements. ${ }^{6}$

Although the theory of energy relaxation of hot electrons in quantum wells or semiconductor superlattices is well developed, ${ }^{7,8}$ the luminescence studies in heterostructures at low temperatures have revealed some information on the relaxation of photocreated carriers which have been omitted in the hot-electron theory. The main relaxation channel of high-energy electrons is related to the emission of optical phonon, ${ }^{8}$ so that the electron-electron and electron-acoustic phonon interaction usually can be ignored. The low-temperature optical studies of heterostructures ${ }^{2}$ with the characteristic spectral spacing much smaller than the optical-phonon frequency reveal another regime of slow relaxation which takes place at the last stage of fast cooling of photocreated carriers. ${ }^{7,8}$ It consists in the relaxation of a very few nonequilibrium electrons which provisionally occupy the first excited subband and produce pronounced recombination, because the overlap of their wave functions with those of spatially separated holes is much better than that of the electrons from the ground subband. ${ }^{2,5}$

The present work was motivated by the studies of the recombination of $2 \mathrm{D}$ electrons in the low-density GaAs$\mathrm{Al}_{x} \mathrm{Ga}_{1-x} \mathrm{As}$ heterostructures with the holes bound at a spatially separated $\delta$-doped acceptor layer. ${ }^{2,5}$ In this case, optical activity of the higher size-quantized subband has been found to be sensitive to the electron lifetime in it. In what follows we study the rate of such an intersubband relaxation in ideally pure heterostructures at low temperature (formally, at $T=0$ ). That is, we consider the degenerate electron gas in the lowest subband with few nonequilibrium carriers in the excited one, so that the problem can be treated as a single-particle one, and the intersubband relaxation rate can be described by the sum of the rates in different open relaxation channels shown in Fig. 1. One of them is formed by the acoustic-phonon emission and will be analyzed in Sec. II. Another mechanism to be considered below is related to the electron-electron scattering and can be viewed as the Auger process involving one nonequilibrium electron from the higher subband and the degenerate Fermi gas in the lowest one. In symmetric quantum wells this process is forbidden by the parity conservation rules, but in (a)

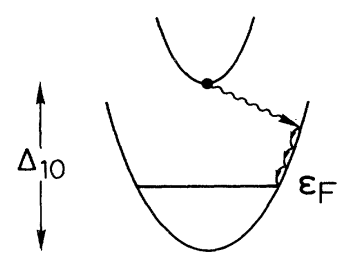

(b)

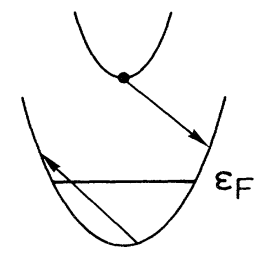

FIG. 1. Intersubband relaxation channels to be considered. (a) The channel of the acoustic-phonon emission is formed by the processes involving phonons with the optimal frequency $s / \lambda_{z}$ due to the intersubband transition selection rules. (b) The Auger-like intersubband relaxation process. $\Delta_{10}$ and $\epsilon_{F}$ are the intersubband splitting and the Fermi energy of 2D electron gas; $\lambda_{z}$ is the effective well width. 
heterostructures the symmetry is broken and the Augertype intersubband relaxation becomes possible. This relaxation channel will be considered in Sec. III.

\section{ELECTRON-PHONON INTERSUBBAND RELAXATION}

The rate of the intersubband relaxation assisted by the acoustic-phonon emission can be calculated according to the general rules, ${ }^{9,10}$

$$
\begin{aligned}
\tau_{\text {phon }}^{-1}=\frac{2 \pi}{\hbar} \int & \frac{d \mathbf{q}}{(2 \pi)^{3}}\left[1-n\left(\hbar q_{\|}\right)\right] \delta\left(\Delta_{10}-\frac{\hbar^{2} q_{\|}^{2}}{2 m}-\hbar s q\right) \\
& \times \hbar \frac{q^{2} \Xi^{2}+\beta^{2}}{2 \mu_{0} s q} F_{10}\left(q_{z}\right),
\end{aligned}
$$

as a sum of partial probabilities of phonon emission with all possible momenta allowed by the momentum and energy conservation law. In Eq. (1) we account for both unscreened ${ }^{11}$ deformation $(\Xi)$ and piezoelectric $(\beta)$ electron-phonon coupling, but do not distinguish the longitudinal and transverse sound velocities $s$, so that $\Xi^{2}$ and $\beta^{2}$ are the effective interaction constants summed over different lattice modes; $\mu_{0}$ is a mass per unit cell, and $m$ is the effective electron mass. In what follows, we scale the relaxation rates in the phonon channel by the effective rates $\tau_{P}^{-1}$ and $\tau_{D}^{-1}$. They describe the relaxation of an electron with the characteristic energy equal to the Debye frequency $\omega_{D}$ due to the piezoelectric and deformation-potential interaction, respectively,

$$
\begin{gathered}
\tau_{P}^{-1}=\frac{\beta^{2}}{2 \pi \hbar \mu_{0} s^{2}} \sqrt{\frac{2 m \omega_{D}}{\hbar}}, \\
\tau_{D}^{-1}=\frac{\Xi^{2}}{2 \pi \hbar \mu_{0} s^{2}}\left(\frac{2 m \omega_{D}}{\hbar}\right)^{3 / 2} .
\end{gathered}
$$

In what follows we present all the results in terms of these effective rates which we shall consider as material parameters describing the electron-phonon interaction constants (i.e., instead of $\Xi$ and $\beta$ ). For reference, in GaAs $\tau_{P} \approx 8 \mathrm{ps}$ and $\tau_{D} \approx 4 \mathrm{ps},{ }^{9}$ and $\hbar \omega_{D} \sim 30 \mathrm{meV}$.

Each partial probability is determined by the electronphonon coupling and by the intersubband matrix element

$$
F_{10}\left(q_{z}\right)=\left|\int d z \varphi_{0}(z) \varphi_{1}(z) \exp \left(i q_{z} z\right)\right|^{2},
$$

which depends on the shape and the width $\lambda_{z}$ of the confining potential in the heterostructure. When one of the potential walls is sharp, the form factor $F_{10}$ has the asymptotic form

$$
F_{10}\left(q_{z}\right)=\left\{\begin{array}{l}
q_{z}^{2}\left|\int \varphi_{0}(z) \varphi_{1}(z) z d z\right|^{2}, \quad q_{z} \rightarrow 0 \\
4\left|\varphi_{0}^{\prime}(0) \varphi_{1}^{\prime}(0)\right|^{2} / q_{z}^{6}, \quad q_{z} \rightarrow \infty
\end{array}\right.
$$

In this equation the limit of small $q_{z}$ is described by the dipole expansion of the transition-matrix element, whereas the expression for the opposite limit [it contains spatial derivatives of the subband wave functions $\varphi_{0}(z)$ and $\varphi_{1}(z)$ near the wall] can be obtained from Eq. (2) after integrating by parts.

Further, in the initial state of the process the electron lies in the bottom of the higher subband (it has the momentum $p_{\|}=0$ along the plane of device) and the ground subband is filled up to the Fermi level $\epsilon_{F}$. Hence, in the final state the relaxed electron cannot have its momentum smaller than $p_{F}$, which produces the finite shift of the phonon-emission threshold as compared to the Fermi-energy crossing with the bottom of the excited subband: $\Delta_{10}-\epsilon_{F}>s p_{F}$. Above the threshold, the phonon momentum across the heteroboundary should be smaller than

$$
q_{c}=\sqrt{\left(\frac{\Delta_{10}-\epsilon_{F}}{\hbar s}\right)^{2}-\frac{p_{F}^{2}}{\hbar^{2}}},
$$

which gives us the upper integration limit in Eq. (1). If we introduce the dimensionless variables $\xi=q_{z} \lambda_{z}$, the rate in Eq. (1) can be rewritten as

$$
\begin{aligned}
\tau_{\mathrm{phon}}^{-1}= & \tau_{P}^{-1} \sqrt{\frac{m s^{2}}{2 \hbar \omega_{D}}} \\
& \times \int_{0}^{q_{c} \lambda_{z}} d \xi \frac{F_{10}(\xi)}{\sqrt{\xi^{2}+\delta_{10}}}\left[1+\frac{\tau_{P}}{\tau_{D}} \frac{1+\xi^{2} / \delta_{10}}{\hbar \omega_{D} / \Delta_{10}}\right],
\end{aligned}
$$

where the parameter $\delta_{10}=2 m \lambda_{z}^{2} \Delta_{10} / \hbar^{2}$ gives the relation between the effective well width $\lambda_{z}$ and intersubband splitting $\Delta_{10}$ and should be specified for each confining potential shape.

(a) Near the threshold the perpendicular phonon momentum component is small, $q_{c} \lambda_{z} \ll \delta_{10}$, and the relaxation rate can be thus derived from Eq. (3) as

$$
\tau_{\text {phon }}^{-1}=\frac{d_{10}}{3} \tau_{P}^{-1} \sqrt{\frac{m s^{2}}{2 \hbar \omega_{D}}}\left(q_{c} \lambda_{z}\right)^{3}\left[1+\delta_{10} \frac{\tau_{P}}{\tau_{D}} \frac{\Delta_{10}}{\hbar \omega_{D}}\right]
$$

where $d_{10}=0.43$ and $\delta_{10}=1.75$ in a triangular well.

(b) When a nonequilibrium electron relaxes in an almost empty well, the energy transferred to the lattice is relatively high and allows the emitted phonon to have momentum which exceeds the inverse quantumwell width. In such a case the emission of high-energy phonons is suppressed because of suppression of the short-wavelength Fourier transform of smooth wave functions $\varphi_{0}$ and $\varphi_{1}$ in Eq. (2), and all the relaxation in this channel is formed by the phonons with optimal frequencies $\sim s / \lambda_{z}$. Hence, we are able to extend the integration in Eq. (3) to the formal infinity and find that

$$
\tau_{\text {phon }}^{-1}=\tau_{P}^{-1} \sqrt{\frac{m s^{2}}{2 \hbar \omega_{D}}}\left[A_{10}+B_{10} \frac{\tau_{P}}{\tau_{D}} \frac{\Delta_{10}}{\hbar \omega_{D}}\right],
$$

where the parameters $A_{10}$ and $B_{10}$ are determined by the shape of the well. In a triangular well, $A_{10}=0.25$, $B_{10}=1.1$. The rate in Eq. (5) describes the fastest possible relaxation in the phonon channel and does not depend strongly on the distinct value of the Fermi energy in $2 \mathrm{D}$ electron gas or on the intersubband splitting. In 
GaAs- $\mathrm{Al}_{x} \mathrm{Ga}_{1-x} \mathrm{As}$ heterostructures it can be estimated as $\tau_{\text {phon }}^{-1} \sim 10^{9} \mathrm{sec}^{-1}$. As one can find after comparing Eqs. (4) and (5), the crossover between regimes (a) and (b) occurs at $q_{c} \lambda_{z} \approx 1.5$, which corresponds to values of $\Delta_{10}-\epsilon_{F}$ below $1 \mathrm{meV}$ in the structures with the 2D electron density in the range of $10^{11}-10^{12} \mathrm{~cm}^{-2}$.

\section{RELAXATION DUE TO INTERSUBBAND ELECTRON-ELECTRON SCATTERING}

Another channel which can compete with the relaxation accompanied by acoustic-phonon emission is related to the electron-electron scattering in the system. The Auger-like process to be considered below is shown in Fig. 1(b). A nonequilibrium electron initially occupies the state in the bottom of the higher subband and then releases its excess energy in a scattering with one of the electrons from the ground subband. After this collision it occupies some state above the Fermi level in the lowest subband. The amplitude of this Auger transition depends on the mutual orientation of spins of electrons involved into the process. When they are of an opposite sign [we shall call this the "spin- $(\uparrow \downarrow)$ " process], the amplitude is simply determined by the matrix element of the Coulomb interaction (which we consider as unscreened ${ }^{11}$ )

$$
\begin{aligned}
M_{\uparrow \downarrow} & =M\left(0, \mathbf{p}+\mathbf{p}^{\prime} ; \mathbf{p}, \mathbf{p}^{\prime}\right) \\
& =\frac{2 e^{2}}{\chi} \int \frac{d q_{z}}{q_{z}^{2}+\mathbf{p}^{2}} F_{10}\left(q_{z}\right) F_{00}^{*}\left(q_{z}\right),
\end{aligned}
$$

whereas the scattering amplitude for two electrons with the same spins ["spin- $(\uparrow \uparrow)$ " process] is composed of two contributions: from the direct and exchange Coulomb interaction, ${ }^{9}$

$$
M_{\uparrow \uparrow}=M\left(0, \mathbf{p}+\mathbf{p}^{\prime} ; \mathbf{p}, \mathbf{p}^{\prime}\right)-M\left(0, \mathbf{p}+\mathbf{p}^{\prime} ; \mathbf{p}^{\prime}, \mathbf{p}\right) .
$$

In Eq. (6) $F_{00}$ is the Fourier transform of a square of the ground subband wave function analogous to that in Eq. (2) and $\chi$ is the dielectric susceptibility of material (in GaAs $\chi \sim 13$, and we do not distinguish between $\chi$ on different sides of the heteroboundary).

The amplitude of the intersubband electron-electron scattering event strongly depends on the shape of a confining potential. When the well is symmetric, the parity of the electronic state is a conserving quantum number, so that the Auger process between the closest subbands is forbidden. In heterostructures the built-in electric field makes the confining potential asymmetric (usually it can be approximated by a triangular well), and the Auger process is, therefore, allowed. After this, the Auger rate takes the form

$$
\begin{gathered}
\tau_{\mathrm{Aug}}^{-1}=\frac{2 \pi}{\hbar}\left(\frac{2 e^{2}}{\chi}\right)^{2} \int \frac{d \mathbf{p} d \mathbf{p}^{\prime}}{(2 \pi)^{4}}[1-n(\mathbf{p})]\left[1-n\left(\mathbf{p}^{\prime}\right)\right] n\left(\mathbf{p}+\mathbf{p}^{\prime}\right) \delta\left(\Delta_{10}-\frac{\hbar^{2}}{m} \mathbf{p p}^{\prime}\right) \\
\times \lambda_{z}^{2}\left[G_{\uparrow \uparrow}\left(\lambda_{z}^{2} p^{2}, \lambda_{z}^{2} p^{\prime 2}\right)+G_{\uparrow \downarrow}\left(\lambda_{z}^{2} p^{2}, \lambda_{z}^{2} p^{\prime 2}\right)\right]
\end{gathered}
$$

where

$$
G_{\uparrow \downarrow}\left(u, u^{\prime}\right)=\left|\int_{-\infty}^{\infty} \frac{d \xi F_{10}(\xi) F_{00}^{*}(\xi)}{\xi^{2}+u}\right|^{2}
$$

and

$$
\begin{aligned}
& G_{\uparrow \uparrow}\left(u, u^{\prime}\right)=\left(u-u^{\prime}\right)^{2} G_{\uparrow \uparrow}^{\prime}\left(u, u^{\prime}\right), \\
& G_{\uparrow \uparrow}^{\prime}\left(u, u^{\prime}\right)=\left|\int \frac{d \xi F_{10}(\xi) F_{00}^{*}(\xi)}{\left(\xi^{2}+u\right)\left(\xi^{2}+u^{\prime}\right)}\right|^{2} .
\end{aligned}
$$

In parametrizing the effective cross sections of spin- $(\uparrow \downarrow)$ and spin- $(\uparrow \uparrow)$ processes in Eqs. (8) and (9) we use the same dimensionless variable $\xi=q_{z} \lambda_{z}$ as in Eq. (3). We also similarly parametrize the electron planar momenta, $u=\lambda_{z}^{2} p^{2}$ and $u^{\prime}=\lambda_{z}^{2} p^{\prime 2}$, which allows us to rewrite Eq. (7) as

$$
\tau_{\text {Aug }}^{-1}=\frac{e^{4} m}{\chi^{2} \hbar^{3}}\left[g_{\uparrow \uparrow}\left(\epsilon_{F} / \Delta_{10}\right)+g_{\uparrow \downarrow}\left(\epsilon_{F} / \Delta_{10}\right)\right] .
$$

The characteristic value of the rate $\tau_{\text {Aug }}^{-1}$ in this expression is scaled by natural units of the donor-scaled Rydberg in the semiconductor, $\mathrm{Ry} / \hbar=e^{4} m / \chi^{2} \hbar^{3}$, whereas the fact that the transition is the intersubband one is taken into account by numerical factors $g_{\uparrow \uparrow}(x)$ and $g_{\uparrow \downarrow}(x)$ deter- mined by the shape of the well and dependent on the Fermi-to-intersubband energy ratio in the structure.

The intersubband electron-electron relaxation process has two obvious thresholds. The first corresponds to the Fermi-energy crossing with the higher subband, when the occupation of the higher subband becomes equilibrium. It occurs at some electronic density $n_{e}^{c}$ at which $\epsilon_{F}\left(n_{e}^{c}\right)=$ $\Delta_{10}\left(n_{e}^{c}\right)$. The second threshold is related to the lowest densities, when the absence of carriers makes the notion of electron-electron scattering meaningless, and in the rest of this section we find the analytical expression of the $\tau_{\text {Aug }}^{-1}$ in these two limits and then estimate it in the region of intermediate densities.

(a) Near the threshold density $n_{e}^{c}$ the energy transferred in the process is negligibly small and in the final state both of the colliding electrons have approximately the same values of their momenta $p \approx p^{\prime} \approx p_{F}$ as an incident electron from the ground subband. The kinematics of this process is shown in Fig. 2(a). A characteristic accessible volume of the phase space of this scattering event is small and proportional to the square of a small deviation of the electron final-state momentum from the Fermi momentum $p_{F}$, which gives

$$
g_{\uparrow \downarrow}\left(\frac{\epsilon_{F}}{\Delta_{10}} \rightarrow 1\right)=\frac{\delta_{10} G_{\uparrow \downarrow}\left(\delta_{10}, \delta_{10}\right)}{(2 \pi)^{2} \sqrt{3}}\left(1-\frac{\epsilon_{F}}{\Delta_{10}}\right)^{2} .
$$


(a)

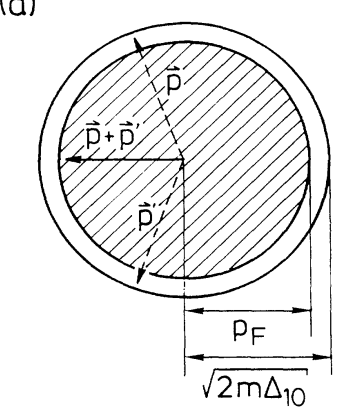

(b)

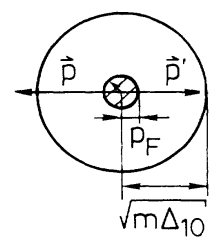

FIG. 2. This figure shows the accessible phase space of the intersubband electron-electron scattering near the Fermi-level crossing with the bottom of the first excited subband (a) and in the case of the almost empty well (b).

The Auger rate in the electron-electron scattering channel with aligned spins is even more suppressed by the cancellation between direct and exchange Coulomb terms in Eq. (9) and results in

$$
g_{\uparrow \uparrow}\left(\frac{\epsilon_{F}}{\Delta_{10}} \rightarrow 1\right)=\frac{\delta_{10}^{3} G_{\uparrow \uparrow}^{\prime}\left(\delta_{10}, \delta_{10}\right)}{6 \sqrt{3}(2 \pi)^{2}}\left(1-\frac{\epsilon_{F}}{\Delta_{10}}\right)^{4} .
$$

To estimate, near the threshold $g_{\uparrow \downarrow} \approx 0.66 \times 10^{-3}(1-$ $\left.\epsilon_{F} / \Delta_{10}\right)^{2}$ and $g_{\uparrow \uparrow} \approx 0.34 \times 10^{-4}\left(1-\epsilon_{F} / \Delta_{10}\right)^{4}$.

(b) The kinematics of the Auger process in almost empty heterostructures is illustrated in Fig. 2(b). When $\epsilon_{F} \ll \Delta_{10}$, the initial-state momenta of both interacting particles are almost zero, whereas in the final state they can be approximated by $p \approx p^{\prime} \approx \sqrt{m \Delta_{10}}$. The probability of the Auger process in the spin- $(\uparrow \downarrow)$ channel is proportional to the effective volume of the phase space $d \mathbf{p} \sim p_{F}^{2}$, and produces the linear dependence

$$
g_{\uparrow \downarrow}\left(\frac{\epsilon_{F}}{\Delta_{10}} \rightarrow 0\right)=\frac{G_{\uparrow \downarrow}\left(\delta_{10} / 2, \delta_{10} / 2\right)}{2 \pi}\left(\frac{\epsilon_{F}}{\Delta_{10}}\right) .
$$

The amplitude of the Auger process in the other channel [see Eq. (9)] contains one additional multiplier $\left(p^{2}-\right.$ $\left.p^{\prime 2}\right)^{2} \sim p_{F}^{2} m \Delta_{10}$ which results from the compensation between the direct and exchange interaction terms. This gives

$$
g_{\uparrow \uparrow}\left(\frac{\epsilon_{F}}{\Delta_{10}} \rightarrow 0\right)=\frac{\delta_{10}^{4} G_{\uparrow \uparrow}^{\prime}\left(\delta_{10} / 2, \delta_{10} / 2\right)}{16 \pi}\left(\frac{\epsilon_{F}}{\Delta_{10}}\right)^{2},
$$

and in a triangular well we can specify $g_{\uparrow \downarrow} \approx 0.83 \times$

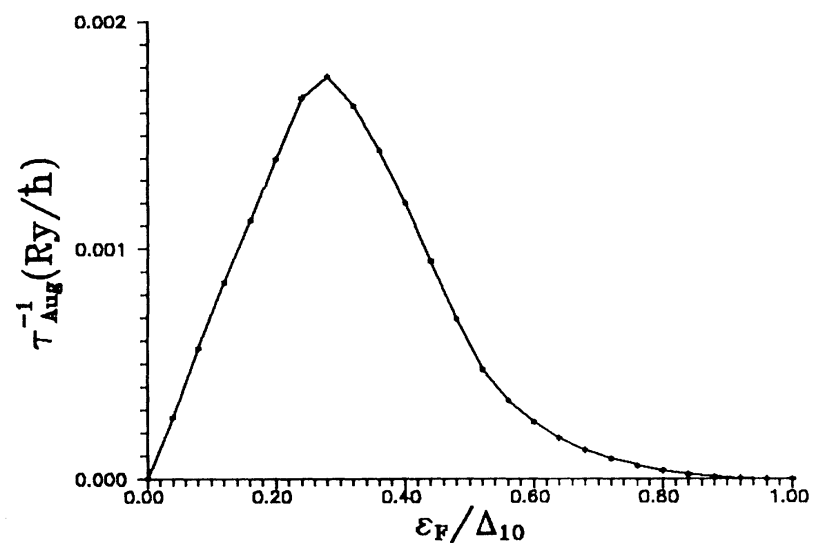

FIG. 3. The Auger rate calculated numerically for the triangular-well model. The rate $\tau_{\text {Aug }}^{-1}$ is measured in the units of the donor-scaled Rydberg in the semiconductor, $\mathrm{Ry} / \hbar=e^{4} m / \chi^{2} \hbar^{3}$. In GaAs Ry/ $\hbar \approx 2 \times 10^{13} \mathrm{sec}^{-1}$.

$10^{-2}\left(\epsilon_{F} / \Delta_{10}\right)$ and $g_{\uparrow \uparrow} \approx 0.24 \times 10^{-2}\left(\epsilon_{F} / \Delta_{10}\right)^{2}$.

(c) The Auger rate in the intermediate density region can be found numerically from Eq. (10), but for an estimation it is enough to analyze the crossover between the asymptotical behavior in two regimes (a) and (b). The crossover occurs at $\epsilon_{F} / \Delta_{10} \sim 0.1$, and near this point the functions $g_{\uparrow \downarrow}$ and $g_{\uparrow \uparrow}$ both reach their maxima which can be estimated as $g_{\uparrow \downarrow}^{\max } \sim 10^{-3}$ and $g_{\uparrow \uparrow}^{\max } \sim 10^{-4}$. This estimation shows that the intersubband scattering of electrons with opposite spins is always dominant and the functional dependence of the Auger rate on $\epsilon_{F}$ calculated numerically for the triangular well (see Fig. 3) coincides with that of $g_{\uparrow \downarrow}\left(\epsilon_{F} / \Delta_{10}\right)$. In GaAs- $\mathrm{Al}_{x} \mathrm{Ga}_{1-x} \mathrm{As}$ heterostructures the maximal Auger rate can be now estimated from Eq. (10) as $4 \times 10^{10} \mathrm{sec}^{-1}$.

\section{CONCLUSIONS}

In summarizing the above analysis, we compare the contributions of the intersubband electron-electron scattering and of acoustic-phonon emission to the intersubband relaxation of nonequilibrium electrons in an ideally pure heterostructure at low temperatures. After taking the Fermi-energy crossing with the bottom of the first excited subband as a reference point, one can see that, first, at lower densities the Auger channel is the main one. But once the emission of a phonon is allowed by the energy conservation law, the electronphonon interaction becomes immediately dominant and provides the relaxation rate of the order of $10^{9} \mathrm{sec}^{-1}$ (this value is obtained for the GaAs- $\mathrm{Al}_{x} \mathrm{Ga}_{1-x} \mathrm{As}$ heterostructure). The latter estimation gives the maximal intersubband relaxation rate one can expect from the acoustic-phonon emission in the triangular-well model. On the other hand, the rate of the Auger process increases with the increase of $\Delta_{10}-\epsilon_{F}$, so that when the $2 \mathrm{D}$ electron density is that which corresponds to the ratio $\epsilon_{F} / \Delta_{10} \approx 0.7-0.8$, the Auger channel becomes dominant 
again. In the triangular-well model the density at which the crossover between the electron-phonon and electronelectron intersubband relaxation mechanisms takes place lies in the range of $(1-3) \times 10^{11} \mathrm{~cm}^{-2}$. At the crossover density (one can reach it, for example, by a weak variation of illumination power ${ }^{2,5}$ ) the lifetime of an electron in the excited subband shortens, which should produce a fast decrease of this subband luminescence intensity. Finally, at $\epsilon_{F} / \Delta_{10} \approx 0.3$ the rate $\tau_{\text {Aug }}^{-1}$ takes its maximal value (in the $\mathrm{GaAs}-\mathrm{Al}_{x} \mathrm{Ga}_{1-x} \mathrm{As}$ system we estimate it as $4 \times 10^{10} \mathrm{sec}^{-1}$ ) which gives us the fastest possible intersubband relaxation in a low-density heterostructure at low temperatures.

\section{ACKNOWLEDGMENTS}

I thank I.V. Kukushkin, I.B. Levinson, M. Potemski, J.C. Maan, and W. Ruhle for useful discussions. I am also grateful to the Alexander von Humboldt Foundation for support during this work and to the NATO Collaborative Research Grant No. 921333 for the possibility of discussions with colleagues from HMFL-MPI.
* On leave from the Theory Department of the Institute of Solid State Physics, Russian Academy of Sciences, Chernogolovka, Moscow district, 142432 Russia.

${ }^{1}$ S. Schmitt-Rink, D.S. Chemla, and D.A.B. Miller, Adv. Phys. 38, 89 (1989).

${ }^{2}$ I.V. Kukushkin, K.v. Klitzing, K. Ploog, and V.B. Timofeev, Phys. Rev. B 40, 7788 (1989).

${ }^{3}$ M.S. Skolnick et al., Phys. Rev. Lett. 58, 2130 (1987).

${ }^{4}$ A.E. Ruckenstein, S. Schmitt-Rink, and R.C. Miller, Phys. Rev. Lett. 56, 504 (1986).

${ }^{5}$ A.J. Turberfield, S.R. Haynes, P.A. Wright, R.A. Ford, R.G. Clark, J.F. Ryan, J.J. Harris, and C.T. Foxon, Phys. Rev. Lett. 65, 637 (1990); V.E. Kirpichev, I.V. Kukushkin, V.B. Timofeev, V.I. Fal'ko, K.v. Klitzing, and K. Ploog, Pis'ma Zh. Eksp. Teor. Fiz. 54, 630 (1991) [JETP Lett. 54, 636 (1991)].

${ }^{6}$ A.F. Dite, I.V. Kukushkin, V.B. Timofeev, A.I. Filin, and K.v. Klitzing, Pis'ma Zh. Eksp. Teor. Fiz. 54, 393 (1991) [JETP Lett. 54, 389 (1991)]; 54, 635 (1991) [54, 642 (1991)].

${ }^{7}$ S.E. Esipov and I.B. Levinson, Adv. Phys. 36, 331 (1987); P.J. Price, J. Appl. Phys. 53, 6863 (1982).

${ }^{8}$ F.A. Riddoch and B.K. Ridley, J. Phys. C 16, 6871 (1983).
${ }^{9}$ V.F. Gantmakher and I.B. Levinson, in Carrier Scattering in Metals and Semiconductors, Modern Problems in Condensed Matter Physics Vol. 19, edited by V.M. Agranovich and A.A. Maradudin (North-Holland, Amsterdam, 1987).

${ }^{10}$ P.J. Price, Ann. Phys. (N.Y.) 133, 217 (1981).

${ }^{11}$ We use the unscreened electron-phonon interaction because in all regimes considered the momentum of emitted phonons (we mean the phonons whose emission gives the dominant contribution to the relaxation process) is great enough. It can be estimated either as $q \sim p_{F}$ (near the threshold) or as $q \sim \lambda_{z}^{-1}>p_{F}$ (when $\epsilon_{F} \ll \Delta_{10}$ ), which provides conditions for negligible screening in most of realistic structures: $q \lambda_{\text {scr }}>1$, where the screening length $\lambda_{\text {scr }}$ in the degenerate 2D electron gas is of the order of the donor-scaled Bohr radius in the semiconductor (the length scale $q^{-1}$ associated with the process is shorter than the screening length). The same can be said about the electronelectron interaction. As shown in Sec. III, in (a) and (b), the momentum $\Delta p$ transferred in the course of electronelectron collisions is always great: $\Delta p \sim p_{F}$ in case (a) or $\Delta p \sim \lambda_{z}^{-1}$ in case (b). Therefore, the unscreened Coulomb interaction should provide a reasonable approximation to calculate the intersubband relaxation rate. 